\title{
TRUTH AND INFINITY1
}

\author{
R. V. B, RUCKER
}

\begin{abstract}
This paper formalizes Gödel's 1946 conjecture that every settheoretic sentence is decidable from the present axioms plus some true axioms of infinity; and we prove a weak variant of this conjecture to be true for every ZF universe. We then make precise the extent to which unbound quantifiers can be taken to range only over ordinals in ZF, obtaining a sort of normal-form theorem. The last section relates these results to the problem of how wide the class of all sets is.
\end{abstract}

1. This paper is based on Gödel's 1946 remarks about the possible termination of the process of finding stronger set theories. Very little has been written about these remarks (see, however, Takeuti [4]); hopefully this paper will provoke further discussion.

In $\$ 2$ we reprint most of the relevant passage from Gödel [1] and attempt to formalize the two conjectures there presented.

$\S 3$ is devoted to a proof of a version of the stronger of these conjectures: that every $\mathrm{ZF}$-sentence is decidable from $\mathrm{ZF}$ plus some true axioms of infinity. We hasten to point out that our proof is possible only because of a very generous interpretation of the notion "axiom of infinity".

In $\S 4$ we use this result to obtain a normal form theorem for ZF-sentences.

In $\S 5$ we assess our results and discuss the import of Gödel's conjecture. The moral that the class of all sets should not be a model of the power-set axiom is drawn.

One of the results of $\$ 3$ was announced in [2]. The proof of the Lemma in $\S 3$ has been corrected at the referee's suggestion.

2. All formulae and sentences are to be in the language of ZF. We will avoid some notational complications by not always maintaining the distinction between a sentence and its Gödel number. If $x$ is a set or class, $\operatorname{Th}(x)$ is the set of all sentences true in the structure $\left\langle x, \varepsilon_{x}\right\rangle . x \vDash \phi\left[a_{1}, \ldots, a_{n}\right]$ means that $a_{1}, \ldots, a_{n} \in x$ and that the sequence $\left\langle a_{1}, \ldots, a_{n}\right\rangle$ (abbreviated as $\vec{a}$ ) satisfies $\phi$ in the structure $\left\langle x, \varepsilon_{x}\right\rangle . D(x)$ is the set of all pairs $\langle\vec{\phi}, \vec{a}\rangle$ such that $x \vDash \phi[\vec{a}]$.

Let us begin by quoting from Gödel [1], where we have italicized a definition and two conjectures:

"It is certainly impossible to give a combinatorial and decidable characteriza-

Presented to the Society, February 15, 1973; received by the editors September 6, 1974 and, in revised form, April 21, 1975.

AMS (MOS) subject classifications (1970). Primary 02K15, 04A10; Secondary 02A05.

Key words and phrases. Gödel's conjecture, axioms of infinity, class of all sets, reflection principle, power-set axiom.

1 This paper was written with the support of a SUNY/Research Foundation grant. 
tion of what an axiom of infinity is but there might exist, e.g. a characterization of the following sort: An axiom of infinity is a proposition which has a certain (decidable) formal structure and which in addition is true. Such a concept of demonstrability might have the required closure property, i.e. the following could be true: Any proof for a set-theoretic theorem in the next higher system above set theory (i.e. any proof involving the concept of truth which I just used) is replaceable by a proof from such an axiom of infinity. It is not impossible that for such a concept of demonstrability some completeness theorem would hold which would say that every proposition expressible in set theory is decidable from the present axioms plus some true assertion about the largeness of the universe of all sets."

Let $M$ be a set large enough to be sufficiently like the class of all sets. Gödel suggests that there is a decidable (say r.e.) set $P I$ of possible axioms of infinity, and that we can take the set of axioms of infinity to be $I=P I \cap T h(M)$.

A possible formulation of Gödel's first conjecture might be:

(G1) For any sentence $\sigma, \mathrm{MK} \cup\left\{\psi^{(V)}: \psi \in I\right\} \vdash \sigma^{(V)}$ iff ZF $\cup I \vdash \sigma$.

The idea behind (G1) is the following. If we have a ZF-definable set $\Sigma \subseteq P I$, and a $\theta$ such that $R_{\theta} \vDash \Sigma$, then the fact that such a $\theta$ exists either (a) adds nothing to our first-order knowledge of $V$, or (b) can be put in the form "there exists a $\theta$ satisfying such and such an axiom of infinity".

It would seem that $(\mathrm{G} 1)$ will hold provided that the existence of a natural model of each ZF-definable subsystem of ZF $\cup I$ is implied by some sentence in $I$.

We can get different versions of (G1) according to what kind of a theory we put over our "class of all sets" $M$. That is, in (G1) MK could be replaced, for instance, by Ackermann's set theory, Takeuti's Nodal Transfinite Type theory, or Reinhardt's system $S^{+}$.

It is easier to formalize and discuss Gödel's second conjecture:

$$
\mathrm{ZF} \cup I \text { is a complete theory. }
$$

3. Clearly (G2) implies (G1).

Under what assumptions on $M$ and $P I$ will $\mathrm{ZF} \cup(P I \cap T h(M))$ be complete?

All we will require of $M$ is that it be a model of ZF. How shall we choose $P I$ ? There are two cautions.

First, $P I$ should contain arbitrarily long sentences. For if there is an upper bound to the length of the sentences in $P I$, then $P I$ and $I$ are finite. If $I$ is finite, then it is equivalent to a single $\mathrm{ZF}$-sentence, and $\operatorname{Con}(\mathrm{ZF} \cup I)$ will be a $\mathrm{ZF}$ sentence as well. Now $\mathrm{ZF} \cup I$ is consistent since it holds in $M$, so by the incompleteness theorem $\mathrm{ZF} \cup I$ does not decide Con $(\mathrm{ZF} \cup I)$-implying that (G2) fails. Note that this argument will not work in the general case where there is no $\mathrm{ZF}$-term representing $I$, for then $\operatorname{Con}(\mathrm{ZF} \cup I)$ is not a $\mathrm{ZF}$-sentence.

Second, $P I$ should not consist only of sentences whose unbound quantifiers range over the ordinals. For such sentences relativize to $L$, and we believe that "There is a measurable cardinal" is an axiom of infinity. 
Definition. $\sigma$ is an $O$-sentence iff for some formula $\phi, \sigma$ is a sentence of the form $\left(Q_{1} \beta_{1}\right) \cdots\left(Q_{n} \beta_{n}\right)\left(\exists R_{\alpha}\right)\left[R_{\alpha} \vDash \phi\left[\beta_{1}, \ldots, \beta_{n}\right]\right]$, where each $Q_{i}$ is an $\forall$ or an $\exists$.

All the sentences normally called axioms of infinity are $O$-sentences. E.g. "there are arbitrarily large inacessible cardinals" $\leftrightarrow(\forall \beta)\left(\exists R_{\alpha}\right)\left[R_{\alpha} \vDash\right.$ "there is an inacessible cardinal larger than $\beta$ "], "there is a measurable cardinal" $\leftrightarrow\left(\exists R_{\alpha}\right)\left[R_{\alpha} \vDash\right.$ "there is a measurable cardinal"], "there is a compact cardinal" $\leftrightarrow\left(\exists \beta_{1}\right)\left(\forall \beta_{2}\right)\left(\exists R_{\alpha}\right)\left[R_{\alpha} \vDash\right.$ " $\beta_{1}$ is a compact cardinal less than $\beta_{2}$ "].

Let $O S$ be the set of $O$-sentences.

THEOREM 1. If $M$ is a model of $Z F$, then $Z F \cup(O S \cap T h(M))$ is complete.

Theorem 1 is a direct consequence of the lemma proved below.

DEFinition. $\psi$ is an $R$-formula iff for some formula $\phi$ with no unbound quantifiers, $\psi$ has the form $\left(Q_{1} R_{\beta_{1}}\right) \cdots\left(Q_{n} R_{\beta_{n}}\right) \phi\left[R_{\beta_{1}}, \ldots, R_{\beta_{n}}\right]$. An $R$-sentence is an $R$-formula which is a sentence.

Lemma. (i) Every $R$-sentence is equivalent in $Z F$ to some $O$-sentence.

(ii) Every $Z F$-sentence is equivalent in $Z F$ to some $R$-sentence.

\section{ProOF.}

$$
\begin{aligned}
& \left(Q_{1} R_{\beta_{1}}\right) \cdots\left(Q_{n} R_{\beta_{n}}\right) \psi\left[R_{\beta_{1}}, \ldots, R_{\beta_{n}}\right] \\
& \leftrightarrow\left(Q_{1} \beta_{1}\right) \cdots\left(Q_{n} \beta_{n}\right)\left(\exists R_{\alpha}\right)\left[R _ { \alpha } \vDash ( \exists v _ { 1 } , \ldots , v _ { n } ) \left[v_{1}=R_{\beta_{1}} \wedge \cdots\right.\right. \\
& \left.\left.\wedge v_{n}=R_{\beta_{n}} \wedge \psi\right]\right],
\end{aligned}
$$

since $\psi$ has no unbound quantifiers.

(ii) If $\sigma$ is a $\mathrm{ZF}$-sentence, then there is a quantifier-free formula $\psi$ such that $\sigma \leftrightarrow\left(Q_{1} v_{1}\right) \cdots\left(Q_{n} v_{n}\right) \psi$. We will be done if we can prove

$$
\begin{aligned}
\left(Q_{1} v_{1}\right) & \cdots\left(Q_{n} v_{n}\right) \psi \\
& \leftrightarrow\left(Q_{1} R_{\beta_{1}}\right) \cdots\left(Q_{n} R_{\beta_{n}}\right)\left(Q_{1} v_{1} \in R_{\beta_{1}}\right) \cdots\left(Q_{n} v_{n} \in R_{\beta_{n}}\right) \psi
\end{aligned}
$$

(*) will follow if we can prove in ZF that for any quantifier free $\phi$,

$$
(\exists x)\left(Q_{1} R_{\beta_{1}}\right) \cdots\left(Q_{n} R_{\beta_{n}}\right)\left(Q_{1} v_{1} \in R_{\beta_{1}}\right) \cdots\left(Q_{n} v_{n} \in R_{\beta_{n}}\right) \phi
$$

$$
\left(\exists R_{\alpha}\right)\left(Q_{1} R_{\beta_{1}}\right) \cdots\left(Q_{n} R_{\beta_{n}}\right)\left(\exists x \in R_{\alpha}\right)\left(Q_{1} v_{1} \in R_{\beta_{1}}\right) \cdots\left(Q_{n} v_{n} \in R_{\beta_{n}}\right) \phi .
$$

(*) follows inductively from $(* *)$ since replacing $\phi$ by $\sim \phi$ and replacing each side of $(* *)$ by its negation yields $(* *)$ with " $\exists$ " replaced by " $\forall$ ".

Now we prove $(* *)$. The idea is that we replace " $(\exists x)$ " by " $\left(\exists R_{\alpha}\right)$ $\left(\exists x \in R_{\alpha}\right)$ " and then move the $\left(\exists x \in R_{\alpha}\right)$ in past the $\left(Q_{i} R_{\beta_{i}}\right)$ one at a time. One can always interchange two existential quantifiers, so the only problem is to show, for any $k$ between 1 and $n$ such that $Q_{k}$ is $\forall$, that if we fix $R_{\alpha}, R_{\beta_{1}}, \ldots, R_{\beta_{k-1}}$ then 


$$
\begin{aligned}
(\exists x \in & \left.R_{\alpha}\right)\left(\forall R_{\beta_{k}}\right) \cdots\left(Q_{n} R_{\beta_{n}}\right) \\
& \left(Q_{1} v_{1} \in R_{\beta_{1}}\right) \cdots\left(\forall v_{k} \in R_{\beta_{k}}\right) \cdots\left(Q_{n} v_{n} \in R_{\beta_{n}}\right) \phi \\
& \leftrightarrow\left(\forall R_{\beta_{k}}\right)\left(\exists x \in R_{\alpha}\right) \cdots\left(Q_{n} R_{\beta_{n}}\right) \\
& \left(Q_{1} v_{1} \in R_{\beta_{1}}\right) \cdots\left(\forall v_{k} \in R_{\beta_{k}}\right) \cdots\left(Q_{n} v_{n} \in R_{\beta_{n}}\right) \phi .
\end{aligned}
$$

The forward implication is obvious. To prove the reverse implication, assume the right-hand side of $(* * *)$. Now for each value of $\beta_{k}$, define the set

$$
\begin{aligned}
s\left(\beta_{k}\right)=\left\{x \in R_{\alpha}:\left(Q_{k+1} R_{\beta_{k+1}}\right) \cdots\left(Q_{n} R_{\beta_{n}}\right)\left(Q_{1} v_{1} \in R_{\beta_{1}}\right)\right. & \\
& \left.\cdots\left(\forall v_{k} \in R_{\beta_{k}}\right) \cdots\left(Q_{n} v_{n} \in R_{\beta_{n}}\right) \phi\right\} .
\end{aligned}
$$

Since we have assumed the right-hand side of $(* * *)$, each $s\left(\beta_{k}\right)$ is nonempty. Note also that $\beta_{k}<\beta_{k}^{\prime} \rightarrow s\left(\beta_{k}\right) \supseteq s\left(\beta_{k}^{\prime}\right)$. This is true since the only appearance of $\beta_{k}$ in the specification condition of $s\left(\beta_{k}\right)$ is in the part " $\left(\forall v_{k} \in R_{\beta_{k}}\right)$ ", so the smaller $\beta_{k}$ is, the easier it is for an $x$ to satisfy the specification condition of $s\left(\beta_{k}\right)$.

Now, if there is an $x \in \cap_{\beta_{k}} s\left(\beta_{k}\right)$, then this $x$ will witness the truth of the left-hand side of $(* * *)$. We can see, however, that this intersection is nonempty, for if it were empty, the map from $R_{\alpha}$ into $O n$ defined by $f^{\prime} x=\left(\mu \beta_{k}\right)\left[x \notin s\left(\beta_{k}\right)\right]$ would be cofinal in $O n$, contradicting the axiom of replacement. In other words, since $\left\{s\left(\beta_{k}\right): \beta_{k} \in O n\right\}$ is a $\supseteq$-sequence of nonempty sets it must be eventually constant, and must thus have nonempty intersection.

So now we have proved $(* * *)$; so we have $(* *),(*)$, and part (ii) of our Lemma.

4. The Lemma has a certain intrinsic interest as a sort of normal form theorem for ZF. One can sharpen it.

THEOREM 2. There is a $\Delta_{2}^{Z F}$ formula $\tau\left(v_{0}, v_{1}\right)$ such that for every $Z F$-sentence $\sigma$ there are integers $s$ and $n$ and $a$ sequence $Q_{1}, \ldots, Q_{n+1}$ of quantifiers such that

$$
Z F \vdash \sigma \leftrightarrow\left(Q_{1} \beta_{1}\right) \cdots\left(Q_{n+1} \beta_{n+1}\right) \tau\left[s,\left\langle\beta_{1}, \ldots, \beta_{n+1}\right\rangle\right] .
$$

Proof. By the Lemma we can assume that $\sigma$ is an $O$-sentence which we abbreviate as $(\vec{Q} \beta)(\exists \alpha)\left[R_{\alpha} \vDash \psi[\vec{\beta}]\right]$.

Note that

$$
\sigma \leftrightarrow\left\{\begin{array}{l}
(\overrightarrow{Q \beta})(\exists \alpha)(\forall r)\left[r \neq R_{\alpha} \vee\left\langle{ }^{\top} \psi, \vec{\beta}\right\rangle \in D(r)\right] \\
(\overrightarrow{Q \beta})(\exists \alpha)(\exists r)\left[r=R_{\alpha} \wedge\left\langle{ }^{\top} \psi, \vec{\beta}\right\rangle \in D(r)\right]
\end{array}\right.
$$

It is not hard to see that $r=R_{\alpha}$ is a $\Delta_{2}^{\mathrm{ZF}}$ predicate of $r$ and $\alpha$. As is wellknown, $\left\langle{ }^{r} \psi, \vec{\beta}\right\rangle \in D(r)$ is a $\Delta_{1}^{\mathrm{ZF}}$ predicate of ${ }^{\top} \psi{ }^{\top}, \beta$ and $r$. Thus we can see that there is a $\Delta_{2}^{\mathrm{ZF}}$ predicate $\tau^{\prime}$ of ${ }^{\top}, \beta$, and $\alpha$ such that $\sigma \leftrightarrow(\overrightarrow{Q \beta})$ $(\exists \alpha) \tau^{\prime}\left[{ }^{r} \psi>, \vec{\beta}, \alpha\right]$.

Viewing " $(\exists \alpha)$ " as “ $\left(Q_{n+1} \beta_{n+1}\right)$ ", we obtain our result.

Note that in Theorem 2, $s, n$, and the sequence $Q_{1}, \ldots, Q_{n+1}$ are recursive in ${ }^{\top} \boldsymbol{\sigma}$. 
5. It could reasonably be argued that Theorem 1 is not an acceptable answer to (G2) since the set of $O$-sentences does not seem to organically embody the principles by which possible axioms of infinity are obtained, e.g. reflection principles, the principle of analogy to $\omega$, considerations of imaginary extensions of the class of all sets, etc. In particular, it does not seem fair to call the $O$-sentence $\left(\exists R_{\alpha}\right)\left[R_{\alpha} \vDash 2^{\aleph_{0}}=\aleph_{1}\right]$ an axiom of infinity.

On the other hand, if we view the class of all sets as the limit of the $R_{\alpha}$ sequence, the basic form of the $O$-sentences seems correct for axioms of infinity-a string of unbounded ordinal quantifiers followed by the assertion of the existence of a partial universe $R_{\alpha}$ satisfying a certain formula with ordinal parameters. The $O$-sentence formulation strikes us as incorrect only after we realize that every $\mathrm{ZF}$-sentence is equivalent to an $O$-sentence.

It may be that for any constructive set PI of possible axioms of infinity, either (i) (G2) holds, but only because every ZF-sentence is provably equivalent to some sentence in $P I$, or (ii) (G2) fails because consideration of the true members of $P I$ leads one to conceive of a true axiom of infinity which is not in PI. The problem could be that the notion "true axiom of infinity" is not reducible to the notion of truth in the class of all sets plus some constructive notion of possible axioms of infinity ... the notion "possible axiom of infinity" may itself be inherently nonconstructive.

It is, however, the case that certain ZF-sentences, e.g. "there is a Mahlo cardinal", become accepted as true axioms of infinity. One can ignore the problem of determining the degree of nonconstructivity in this acception process and argue that since every ZF-sentence will eventually be either accepted or rejected as a true axiom of infinity, the set of all true axioms of infinity therefore exists and (G2) has a definite meaning. Should (G2) hold for this naive notion of true axiom of infinity? If every set is ordinally definable (it may be significant that this notion was first introduced in [1]), then it might be possible for axioms of infinity to determine the full structure of the universe (see $[3, \S 4]$ for a weak example).

One could perhaps go so far as to compare the (G2) assertion that knowledge about "everything" is reducible to knowledge about "infinity", and the $\mathrm{CH}$ assertion that the class of all hereditarily countable sets can be injected into the class of all hereditarily countable ordinals.

In Theorem 1 of this paper we showed that a weak form of (G2) holds for every model of ZF; and in Theorem 2 we showed that a consequence of Theorem 1 is that in models of ZF, set theory approaches ordinal theory. A viewpoint which we have advocated in [3] is that the process of approximating the width of the universe (e.g. finding new reals) should be as interminable as the process of approximating the length of the universe (e.g. adding more ordinals). It seems that if we wish to emphasize the width of the universe, then we cannot expect any form of (G2) to hold.

The key fact in the proof of Theorem 1 is that in $\mathrm{ZF}$ the universe is the union of an increasing ordinal-indexed sequence of sets, the $R_{\alpha}$ sequence; and this fact is basically a consequence of the power-set axiom. The point we wish to make here is that $Z F^{-}$(i.e. $Z F$ set theory without the power-set axiom) is an essentially richer theory about the class of all sets than $Z F$.

In $\mathrm{ZF}^{-}$one might replace $O$-sentences by $o$-sentences, where $\sigma$ is an $o$ - 
sentence iff $\sigma$ has the form $(\overrightarrow{Q \beta})(\exists x)[x \vDash \phi[\vec{\beta}]]$ for some formula $\phi$. It does not seem likely that Theorem 1 can be proved with $\mathrm{ZF}$ and $O S$ replaced by $\mathrm{ZF}^{-}$and $o S$. Nor does it seem likely that Theorem 2 holds for $\mathrm{ZF}^{-}$.

\section{REFERENCES}

1. K. Gödel, Remarks before the Princeton Bicentennial Conference on Problems in Mathematics, 1946, The Undecidable, M. Davis (editor), Raven Press, Hewlett, N.Y., 1965, pp. 84-88.

2. R. Rucker, Truth and infinity, Notices Amer. Math. Soc. 20 (1973), A-444. Abstract \# 73TE45.

3. - On Cantor's continuum problem (to appear).

4. G. Takeuti, Formalization principle, Logic, Methodology and Philos., III (Proc. Third Internat. Congr., Amsterdam, 1967), North-Holland, Amsterdam, 1968, pp. 105-118. MR 40 \#5441.

Department of Mathematics, State University College at Geneseo, Geneseo, New York 14454 